\title{
AN IN SITU STUDY OF RECONSTRUCTED GOLD ELECTRODE SURFACES BY SECOND HARMONIC GENERATION
}

\author{
A. FRIEDRICH, B. PETTINGER, D.M. KOLB \\ Fritz-Haber-Institut der Max-Planck-Gesellschaft, Faradayweg 4-6, D-1000 Berlin 33, Germany \\ G. LÜPKE, R. STEINHOFF and G. MAROWSKY \\ Max-Planck-Institut Jür Biophysikalische Chemie, Am Fassberg, D-3400 Göttingen, Federal Republic of Germany
}

Received 20 July 1989; in final form 30 August 1989

Second harmonic generation (SHG) was employed to monitor in situ the potential-induced reconstruction of $\mathrm{Au}(111)$ and $\mathrm{Au}(100)$ electrodes. Rotating the sample by $360^{\circ}$ about the surface normal yields for the unreconstructed $\mathrm{Au}(111)-(1 \times 1)$ surface the well-known threefold symmetry pattern in the SHG intensity, while Au(100)-(1 $\times 1)$ shows no rotational anisotropy, as expected for $\mathrm{C}_{4 \mathrm{v}}$ symmetry. For the reconstructed $\mathrm{Au}(111)-(1 \times 23)$ surface, however, an additional onefold symmetry pattern is observed, which allows in situ monitoring of the structural transition between $(1 \times 1)$ and $(1 \times 23)$. For the reconstructed Au $(100)-(5 \times 20)$ surface, a threefold symmetry pattern was found.

\section{Introduction}

Structure determinations for electrode surfaces, preferably in situ and on a microscopic level, are still one of the most important challenges in physical electrochemistry [1]. A number of techniques are now available for the study of surfaces in an electrochemical cell, including electroreflectance spectroscopy in the VIS-near UV range [2], EXAFS [3] or grazing incidence X-ray diffraction [4] and scanning tunneling microscopy $[5,6]$, all of which have advanced our knowledge on electrode-surface structures and their change with electrochemical manipulation. For the study of surface symmetries, which becomes increasingly important with the use of single-crystal material in electrochemistry and which plays a crucial role in surface-reconstruction effects, a truly structurc-sensitive method for routinc use, like for example LEED in UHV studies, is not yet available, because ordinary (linear) optical spectroscopies such as electroreflectance lack structure sensitivity, while the more structure-specific X-ray tcchniques are still prohibitivcly claboratc for ordinary applications, as they require synchrotron radiation. In this respect, second harmonic generation
(SHG) shows promise for the in situ investigation of single-crystal surface structures. In a pioneering study, Richmond et al. have demonstrated that the threefold symmetry of a $\mathrm{Ag}(111)$ surface can be clearly detected in situ by measuring the rotational anisotropy of its SHG signal [7]. This is done by recording the SHG intensity as a function of azimuthal angle as the sample is rotated about its surface normal. For a (111) surface of an fcc metal and for ppolarization three peaks, each $120^{\circ}$ apart, are found $[7,8]$. Analogous studies using linear optics revealed the twofold symmetry of a $\mathrm{Ag}(110)$ surface [9] but not the threefold symmetry of $\mathrm{Ag}(111)$, as lincar spectroscopy can only detect rotational symmetries of $\mathrm{C}_{1}$ or $\mathrm{C}_{2}$. The apparently higher structure sensitivity of nonlinear optical effects could make SHG a powerful tool for in situ studies, especially in the casc of surface reconstruction, where the surface atoms of a solid rearrange themselves in a structure different from that expected from a mere termination of the bulk.

Recently we have focused our attention on the reconstruction of the threc low-index faces of gold single crystals [ $10-12]$, which are known to reconstruct in UHV when they are clean. By applying a wide va- 
ricty of techniques ranging from classical electrochemical methods, such as cyclic voltammetry. to electroreflectance spectroscopy and ex situ electron diffraction (LEED and RHEED), we have established that reconstructed gold surfaces are stable in contact with an aqueous solution, if certain potential requirements are met $[10-12]$. Furthermore, for $\mathrm{Au}(100)$ and $\mathrm{Au}(111)$ a potential-induced reconstruction was observed, i.e. after the reconstruction was lifted by the adsorption of anions, the reconstructed surface could be regenerated simply by applying appropriate electrode potentials [11,12].

These surfaces are, therefore, ideally suited to test the structure sensitivity of SHG. In the following, we present an in situ SHG investigation of gold electrodes, which shows the influence of surface reconstruction on the rotational anisotropy and demonstrates the power of SHG in assessing the stability range of reconstructed surfaces and the kinetics of structural transitions.

\section{Experimental}

The experimental apparatus used in this study consisted of a standard SHG arrangement [13] and conventional electrochemical equipment. The two gold electrodes, single-crystal discs $8 \mathrm{~mm}$ in diameter and $2 \mathrm{~mm}$ thick with surface orientations of (111) and (100), were initially prepared by mechanical polishing and electropolishing and then repeatedly subjected to flame treatment [14], where the electrode is held in a Bunsen flame at $800^{\circ} \mathrm{C}$ for about $3 \mathrm{~s}$ and subsequently quenched in triply distilled water. This flame treatment, which was carried out on the gold electrode before each SHG measurement, has been shown to yield reconstructed surfaces of high quality for $\mathrm{Au}$ (111) and $\mathrm{Au}(100)$ [10]. In order to maintain surface reconstruction in the electrochemical cell, the electrode had to be immersed under potentiostatic control with a fairly negative potential applied (e.g. $-0.4 \mathrm{~V}$ versus SCE for $\mathrm{Au}(100)-(5 \times 20))$. The electrolytes, aqueous solutions of $0.01 \mathrm{M} \mathrm{H}_{2} \mathrm{SO}_{4}$ and $0.01 \mathrm{M} \mathrm{HClO}_{4}$ made from Merck suprapure chemicals and triply distilled water, were deaerated with purified nitrogen. All potentials are quoted with respect to the saturated calomel electrode (SCE). High quality polarization ro- tators and polarizers determined the experimental polarization conditions $(\mathrm{p} / \mathrm{p}, \mathrm{s} / \mathrm{s}$ or $\mathrm{p} / \mathrm{s})$ for incident $(\omega)$ and reflected $(2 \omega)$ beams. In order to improve the signal-to-noise ratio, a gated integrator with a time constant of $8 \mathrm{~s}$ was used. The Nd-YAG laser producing $10 \mathrm{~ns}$ pulses with an energy of $20 \mathrm{~mJ}$ at a repetition rate of $10 \mathrm{~Hz}$ was slightly focused onto the probe at an angle of incidence of $45^{\circ}$ leading to an illuminated area of about $1 \mathrm{~mm}^{2}$. The rotational anisotropy of the SHG intensity was monitored by rotating the gold electrode about its surface normal in the electrochemical cell for fixed polarization conditions for the incident fundamental $(1064 \mathrm{~nm})$ and generated second harmonic $(532 \mathrm{~nm})$ beam.

\section{Results}

\subsection{The Au(111) surface}

In fig. 1, the rotational anisotropy of $\mathrm{Au}(111)$ at two different electrode potentials is shown. Curve (a) corresponds to a freshly prepared (flame-treated) surface which was immersed at $-0.4 \mathrm{~V}$ versus SCE, and the SHG measurement performed at $-0.1 \mathrm{~V}$ versus SCE. Under such conditions, the Au(111) surface is reconstructed into the $(1 \times 23)$ structure, where the surface atoms show a $4 \%$ compression in the [110] direction. Curve (b), on the other hand, has been recorded after the potential was scanned to $U=+0.7 \mathrm{~V}$ versus SCE, where anion adsorption has lifted the reconstruction [10]. While in the latter case the threefold symmetry of the unreconstructed $\mathrm{Au}(111)-(1 \times 1)$ surface is clearly reflected in its rotational anisotropy exhibiting six intensity maxima of about equal height, the corresponding pattern for the $\mathrm{Au}(111)-(1 \times 23)$ surface (curve (a)) is distinctly different. A pronounced onefold symmetry pattern is superimposed on to the threefold one, with two dominant peaks at $90^{\circ}$ and $270^{\circ}$ and the other four peaks markedly reduced in intensity (note that the SHG pattern of fig. la represents a mixture of onefold and threefold symmetry elements: $I_{\text {SHG }}$ $\left.\approx(a \cos \phi+b \cos 3 \phi)^{2}\right)$. Comparison of curves (a) and (b) in fig. 1 demonstrates that the surface structural transition between $(1 \times 1)$ and $(1 \times 23)$ is clearly seen in the rotational anisotropy of the SHG signal, although there is only a $4 \%$ compression of 


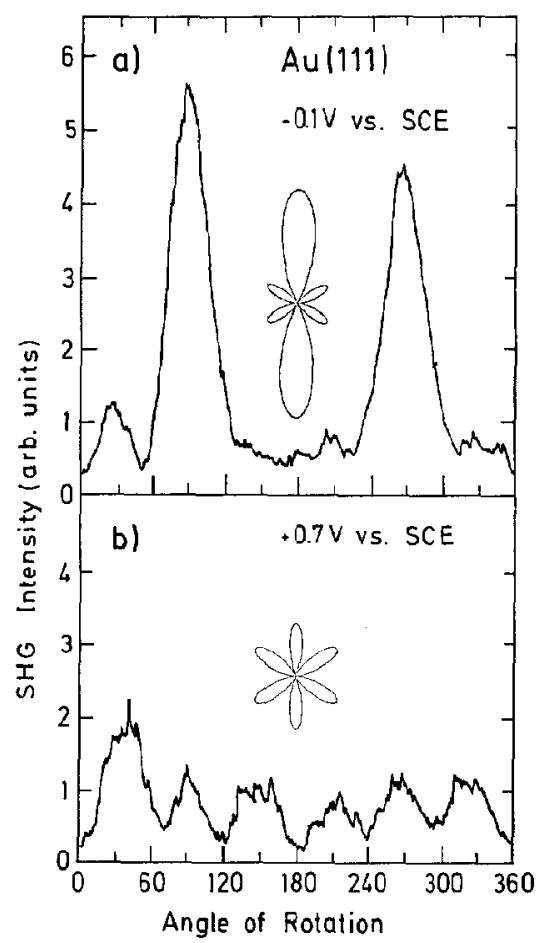

Fig. 1. Second harmonic intensity as a function of angle of rotation for $\mathrm{Au}$ (111) in $0.01 \mathrm{M} \mathrm{H}_{2} \mathrm{SO}_{4}$ at (a) $-0.1 \mathrm{~V}$ and (b) +0.7 $\mathrm{V}$ versus SCE. s-polarized excitation and s-polarized SHG detection in both cases. For illustration purposes the calculated polar plots of the rotational anisotropies for s/s-polarization of a threefold-symmetry surface (a) with and (b) without a onefold symmetry superimposed are also shown.

the top layer in the latter case. This enables us to monitor in situ the structural transition by recording the $\mathrm{SHG}$ intensity at either $90^{\circ}$ or $270^{\circ}$ azimuthal angle as a function of electrode potential. The potential dependence of the SHG signal at $90^{\circ}$ (fig. 2) reveals that in $0.01 \mathrm{M} \mathrm{HClO}_{4}$ the onefold symmetry pattern rapidly decreases for potentials more positive than $+0.4 \mathrm{~V}$, indicating that the $(1 \times 23)$ reconstruction is stable only up to that value, whereas no cathodic limit for the stability range of the $(1 \times 23)$ structure was found. For $U>+0.7 \mathrm{~V}$, the $(1 \times 23) \rightarrow(1 \times 1)$ transition is completed and the $\mathrm{Au}(111)$ surface is now unreconstructed. Scanning the electrode potential back in the reverse direction shows that this structural transition is fully reversible, as the reappearance of the onefold symmetry indicates restoring of the $(1 \times 23)$ structure at negative surface charges (note that the potential of zero charge

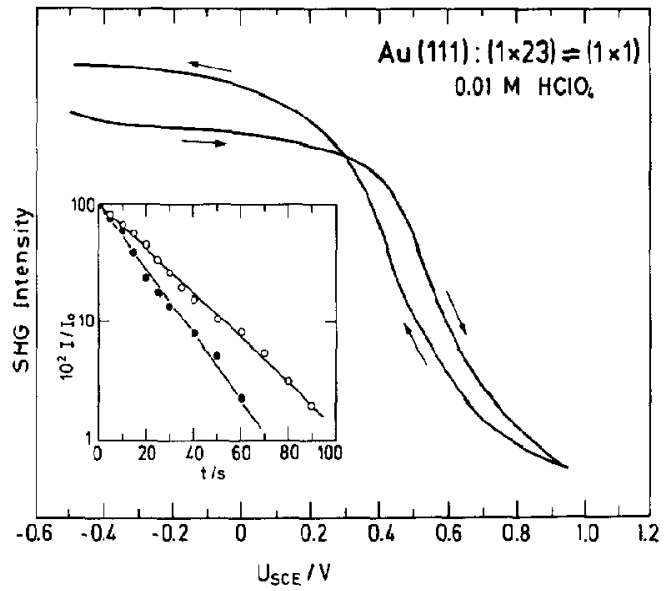

Fig. 2. Second harmonic intensity for $\mathrm{Au}(111)$ in $0.01 \mathrm{M} \mathrm{HClO}_{4}$ as a function of electrode potential, showing the structural transition between $(1 \times 23)$ and $(1 \times 1)$. The azimuthal angle of rotation is $90^{\circ}$ (see fig. 1). s/s-polarization. Scan rate: $1 \mathrm{mV} \mathrm{s}^{-1}$. Insert: time dependence of the normalized SHG intensity after a potential step from -0.2 to $+0.8 \mathrm{~V}$ versus SCE (open circles) and from +0.8 to $-0.2 \mathrm{~V}$ versus SCE (full circles).

for $\mathrm{Au}(111)$ is around $+0.3 \mathrm{~V}$ versus SCE [10]). In order to gain some insight into the transition $\mathrm{ki}$ netics, potential steps between -0.2 and $+0.8 \mathrm{~V}$ versus SCE were employed, and the change in SHG intensity at an azimuthal angle of $90^{\circ}$ followed as a function of time. The transient response shows firstorder kinetics for both cathodic and anodic potential steps (see insert in fig. 2). While for the lifting of the $(1 \times 23)$ reconstruction (anodic step) a decay time $\tau_{1 / 2}=22.7 \mathrm{~s}$ was derived, the subsequent $(1 \times 1)$ $\rightarrow(1 \times 23)$ transition (cathodic step) was found to be even slightly faster with $\tau_{1 / 2}=15.3 \mathrm{~s}$.

\subsection{The $A$ u(100) surface}

Previous studies of $\mathrm{Au}(100)$ electrodes with LEED and RHEED have shown that the reconstructed $\mathrm{Au}(100)-(5 \times 20)$ surface is stable only in a potential range where no anion adsorption takes place [10]. The latter removes reconstruction, i.e. in the presence of adsorbed anions the $\mathrm{Au}(100)$ surface is transformed into the $(1 \times 1)$ structure. However, at negative potentials (e.g. at $-0.4 \mathrm{~V}$ versus $\mathrm{SCE}$ ), the $(5 \times 20)$ structure is regenerated at room temperature due to a potential-induced reconstruction [11]. In order to test the structure sensitivity of SHG in 
the case of $\mathrm{Au}(100)$, the rotational anisotropy was measured for reconstructed and unreconstructed $\mathrm{Au}(100)$ electrodes in $0.01 \mathrm{M} \mathrm{HClO}_{4}$. In fig. 3a a rotational anisotropy of a freshly prepared $\mathrm{Au}(100)$ $(5 \times 20)$ surface at $U=-0.1 \mathrm{~V}$ is shown for p-polarized excitation and p-polarized SIIG detection. Three intensity maxima, about $120^{\circ}$ apart, are clearly seen for the reconstructed surface. Scanning the potential up to $+0.9 \mathrm{~V}$ causes the reconstruction to disappear due to the specific adsorption of sulfate ions, and indeed, the three peaks ascribed to the $(5 \times 20)$ structure are no longer visible (curve (b)). (The intensity variation which is still seen in curve (b) is due to an imperfect alignment of the gold sample causing the surface to wobble during rotation. For the elimination of such effects in the measurement of the reconstructed surface, curve (b) was subtracted from curve (a); see insert in fig. 3.) Stepping the potential back to $-0.2 \mathrm{~V}$ and waiting for several minutes causes the threefold intensity pattern to

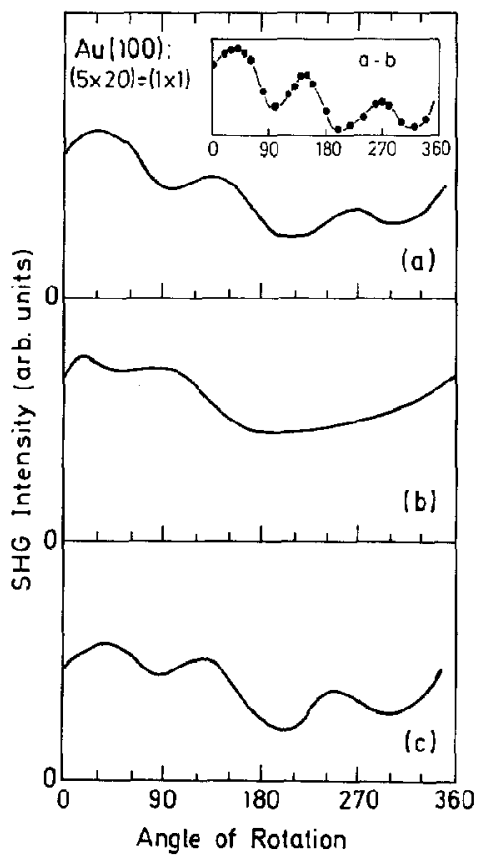

Fig. 3. Second harmonic intensity as a function of angle of rolation for $\mathrm{Au}(100)$ in $0.01 \mathrm{M} \mathrm{HClO}_{4}$ at (a) $-0.1 \mathrm{~V}$ (the surface is reconstructed), (b) $+0.9 \mathrm{~V}$ (reconstruction is lifted), and (c) $-0.2 \mathrm{~V}$ (the surface is reconstructed again). $\mathrm{p} / \mathrm{p}$-polarization. The insert shows the difference between curves (a) and (b), which corresponds to curve (a) corrected for effects due to wobbling of the surface during rotation. reappear, which strongly suggests that the $A u$ (100) surface is reconstructed again under such conditions, in full agreement with earlier observations [11,12].

\section{Discussion}

The above results for $\mathrm{Au}$ demonstrate the great sensitivity of SHG to the structure of the topmost layer. Thus, surface reconstruction effects are readily seen in the rotational anisotropy. For a centrosymmetric solid such as Au, SHG from the bulk vanishes in the dipole approximation [15], whereas for its surface, significant isotropic and anisotropic SHG intensities may be obtained $[8,16]$, which are described by a susceptibility tensor of third rank:

$\mathbf{P}(r, t)=\chi^{(2)}: \mathbf{E}(r, t) \mathbf{E}(r, t)$.

The symmetry of a surface is reflected by the analogous symmetry of the corresponding $\chi^{(2)}$ tensor, and an analysis of this tensor upon azimuthal rotation reveals that at most a threefold symmetry can be resolved using SHG [17]. The six maxima in the SHG intensity as a function of angle of rotation for $\mathrm{s} / \mathrm{s}$ polarization conditions, which are found for the unreconstructed $\mathrm{Au}$ (111) surface (fig. $1 \mathrm{~b}$ ), reflect the well-known $C_{3 v}$ symmetry of this surface. The observation of a threefold symmetry in the SHG experiment for a hexagonal close packed layer implies that - similar to LEED - the second layer has to be involved in the symmetry considerations for the surface [17]. Reconstruction of the Au(111) surface into $(1 \times 23)$, which represents a hexagonal close packed structure with an uniaxial compression of about $4 \%$ in the [110] direction, leads to a $C_{s}$ symmetry superimposed onto the $C_{3 v}$ symmetry. The $(1 \times 23)$ superstructure alone is expected to have $C_{2 v}$ symmetry, but taking the second layer of $\mathrm{Au}(111)$ into account reduces the symmetry for the reconstructed surface to $C_{s}$. Strong support for the $C_{s}$ symmetry of $A u(111)-(1 \times 23)$ is also obtained from the observation of a combination of one-, two- and threefold symmetry patterns, which are found under $\mathrm{p} / \mathrm{s}$ polarization conditions. A more detailed discussion of the SHG tensor in the case of $(1 \times 23)$ reconstruction will be given elsewhere [18].

The very pronounced onefold symmetry of the $(1 \times 23)$ structure is a striking observation, in view 
of the fact that the reconstructed surface consists of domains which should be equally distributed in all three [112] directions. Hence, one would expect the SHG contributions for the onefold symmetry to vanish by interference. From our experimental results we arc led to the conclusion that the various $(1 \times 23)$ domains are not equally distributed, but have a preferential orientation which could be introduced by, e.g. atomic steps. A second point concerning the marked intensity of the $C_{s}$ symmetry may be worth mentioning. As statcd above, the unreconstructed top layer of $\mathrm{Au}$ (111) exhibits $\mathrm{C}_{6 \mathrm{v}}$ symmetry, which cannot be resolved by SHG. Only when taking the first two atomic layers into account does a $\mathrm{C}_{3 \mathrm{v}}$ symmetry emerge; however, such a surface layer possesses some elements of an inversion symmetry and, hence, the SHG intensity for the Au(111)-(1×1) surface may be reduced significantly. On the other hand, SHG is fully allowed for the reconstructed top layer, and consequently, the $(1 \times 23)$ structure with its low rotational symmetry can give rise to a comparatively large SHG signal. Similarly, Heinz et al. [19] have clearly seen in SHG the $(2 \times 1)$ reconstruction of $\mathrm{Si}(111)$ and they were able to monitor the thermally-induced $(2 \times 1) \rightarrow(7 \times 7)$ transition under UHV condilions. Since the $(2 \times 1)$ surface consists - like the $(1 \times 23)$ surface of $\mathrm{Au}(111)$ - of domains distributed among the three $[211]$ directions, we conclude that it is indeed possible to detect symmetry changes by SHG because of unequal distribution of domains.

Similar to $\mathrm{Au}(111)$, the surface reconstruction of $\mathrm{Au}(100)$ can also be seen in SHG experiments. In the latter case, the reconstructed surface consists of a slightly buckled hexagonal close packed top layer, which gives rise to a rather complicated $(5 \times 20)$ LEED pattern [20]. While for the unreconstructed $\mathrm{Au}(100)$ surface with its $\mathrm{C}_{4 \mathrm{v}}$ symmetry the SHG intensity should not show any variation with sample rotation (if a variation of the SHG signal with angle of rotation is observed, it is caused by sample misalignment, see fig. 3b), a threefold symmetry pattern is clearly observed for the reconstructed $\mathrm{Au}(100)$ surface under $p / p$-polarization conditions. We tentatively suggest that this complicated superstructure contains elements of a threefold symmetry, in addition to others which are not readily discernible in this type of measurement. Further experiments are in progress to firmly establish the symmetry properties of the $\chi^{(2)}$ tensor in the case of $\mathrm{Au}(100)$ $(5 \times 20)$.

\section{Conclusions}

Surface reconstruction of Au was used to demonstrate the power of SHG for in situ structural studies and for monitoring electrochemically induced changcs in surface structures. For the rcconstructed $\mathrm{Au}(111)-(1 \times 23)$ surface, a pronounced onefold symmetry pattern was observed in the SHG intensity as a function of angle of rotation which allows one to investigate in great detail surface-structural transitions. In $0.01 \mathrm{M} \mathrm{IIClO}_{4}$ the $(1 \times 23)$ structure was found to be stable up to $+0.4 \mathrm{~V}$ versus SCE, while at more positive potentials anion adsorption causes the reconstruction to disappear. Negative charging of the gold surface fully restores the $(1 \times 23)$ structure. Both transitions, adsorbate-induced lifting and potential-induced restoration of the $(1 \times 23)$ structure, follow first-order kinetics with decay times on the order of 15 to $20 \mathrm{~s}$. In similar experiments, the anion-induced lifting of the $\mathrm{Au}(100)-(5 \times 20)$ structure and the potential-induced reconstruction of $\mathrm{Au}(100)-(1 \times 1)$ were studied by SHG, confirming earlier results obtained by electroreflectance and LEED measurements [10-12].

\section{References}

[1] D.M. Kolb, Ber. Bunsenges. Physik. Chem. 92 (1988) 1175.

[2] D.M. Kolb, in: Spectroelectrochemistry, theory and practice, ed. R.J. Gale (Plenum Press, New York, 1988) ch. 4.

[3] O.R. Melroy, M.G. Samant, G.L. Borges, J.G. Gordon, L. Blum, J.H. White, M.J. Albarelli, M. McMillan and H.D. Albruña, Langmuir 4 (1988) 728.

[4] M.G. Samant, M.F. Toney, G.L. Borges, L. Blum and O.R. Melroy, J. Phys. Chem. 92 (1988) 220.

[5] A.A. Gewirth and A.J. Bard, J. Phys. Chem. 92 (1988) 5563.

[6] J. Wiechers, T. Twomey, D.M. Kolb and R.J. Behm, J. Electroanal. Chem. 248 (1988) 451.

[7] V.L. Shannon, D.A. Koos and G.L. Richmond, J. Chem. Phys. 87 (1987) 1440.

[8] G.L. Richmond, J.M. Robinson and V.L. Shannon, Progr. Surface Sci. 28 (1988) 1.

[9] T.E. Furtak and D.W. Lynch, J. Electroanal. Chem. 79 (1977) 1;

D.M. Kolb, J. Phys. (Paris) 44 (1983) C10-137. 
[10] D.M. Kolb and J. Schneider, Electrochim. Acta 31 (1986) 929.

[11] J. Schneider and D.M. Kolb, Surface Sci. 193 (1988) 579.

[12] M.S. Zei, G. Lehmpguhl and D.M. Kolb, Surface Sci. 221 (1989) 23

[13] V.L. Shannon, D.A. Koos and G.L. Richmond, Appl. Opt. 26 (1987) 3579.

[14] J. Clavilier, R. Faure, G. Guinet and R. Durand, J. Electroanal. Chem. 107 (1980) 205.

[15] Y.R. Shen, The principles of nonlinear optics (Wiley, New York, 1984).
[16] P. Guyot-Sionnest, W. Chen and Y.R. Shen, Phys. Rev. B 33 (1986) 8254.

[17] J.E. Sipe, D.J. Moss and H.M. van Driel, Phys. Rev. B 35 (1987) 1129.

[18] G. Marowsky, G. Lupke, R. Steinhoff, A. Friedrich, B. Pettinger and D.M. Kolb, to be published.

[19] T.F. Heinz, M.T. Loy and W.A. Thompson, Phys, Rev. Letters 54 (1985) 63 .

[20] M.A. Van Hove, R.J. Koesiner, P.C. Stair, J.P. Biberian, L.L. Kesmodel, I. Bartos and G.A. Somorjai, Surface Sci. $103(1981) 189,218$. 